Mitigation and control of the overcuring effect in mask projection microstereolithography

Paul F. O'Neill, Nigel Kent, and Dermot Brabazon

Citation: AIP Conference Proceedings 1896, 200012 (2017); doi: 10.1063/1.5008249

View online: http://dx.doi.org/10.1063/1.5008249

View Table of Contents: http://aip.scitation.org/toc/apc/1896/1

Published by the American Institute of Physics 


\title{
Mitigation and Control of the Overcuring Effect in Mask Projection Micro-Stereolithography
}

\author{
Paul F. O’Neill ${ }^{1, \text { a) }}$, Nigel Kent ${ }^{1,2}$ and Dermot Brabazon ${ }^{1}$ \\ ${ }^{1}$ Advanced Processing Technology Research Centre, Dublin City University, Ireland \\ ${ }^{2}$ School of Mechanical and Design Engineering, Dublin Institute of Technology, Ireland \\ a)Corresponding author: paul.oneill37@mail.dcu.ie
}

\begin{abstract}
Mask Projection micro-Stereolithography (MP $\mu \mathrm{SL})$ is an additive manufacturing technique capable of producing solid parts with micron-scale resolution from a vat of photocurable liquid polymer resin. Although the physical mechanism remains the same, the process differs from traditional laser-galvanometer based stereolithography (SL) in its use of a dynamic mask UV projector, or digital light processor (DLP), which cures each location within each 3D layer at the same time. One area where MP $\mu$ SL has garnered considerable attention is in the field of microfluidics and Lab-on-a-Chip, where complex multistep microfabrication techniques adopted from the semiconductor industry are still widely used, and where MP $\mu$ SL offers the ability to fabricate completely encapsulated fluidic channels in a single step and at low cost [1-3]. However, a significant obstacle exists in the prevention of channel blockage due to overcuring of the polymer resin $[4,5]$. Overcuring can be attributed to the so-called 'back side effect' [2] which occurs during the build process as light from successive layers penetrates into the resin to a depth greater than the layer thickness. This effect is most prevalent in channels or features oriented horizontally (in a parallel plane to that of the build platform). Currently there are two main approaches in controlling the cure depth; 1. the chemical approach, which involves doping the resin material with a chemical light absorber [6-8]; and 2. by improving the system's hardware and optical elements to improve the homogeneity of the light dosage and control the cure depth [9]. Here we investigate a third approach through modification of the 3D CAD file prior to printing to mitigate for UV light leakage from successive build layers. Although used here in conjunction with the MP $\mu S L$ technique, this approach can be applied to a range of SL techniques to improve printer resolution and enable production of internal features with higher dimensional accuracy.
\end{abstract}

\section{INTRODUCTION}

Recently, 3D printing has become a hot topic in the field of microfluidics with a number of groups beginning to adopt it as a method to fabricate fluidic devices $[1,2,10]$. In this respect Stereolithography (SL) is of particular interest, as it enables rapid production of milli- and micro-scale fluidically sealed channels at low cost. However, the SL process is not without its drawbacks, polymer-chemical compatibility and the limitations of the overcuring effect are two of the main barriers to microfluidic fabrication [2]. In this paper we examine one of these drawbacks, the overcuring effect, and implement a simulation based on a mathematical model previously developed by Gong et al. [7]. To understand the limitations of the overcuring effect we must first examine the SL 3D printing process. The basic SL Digital Light Processing (DLP) process is outlined below in Fig 1. 
(a)

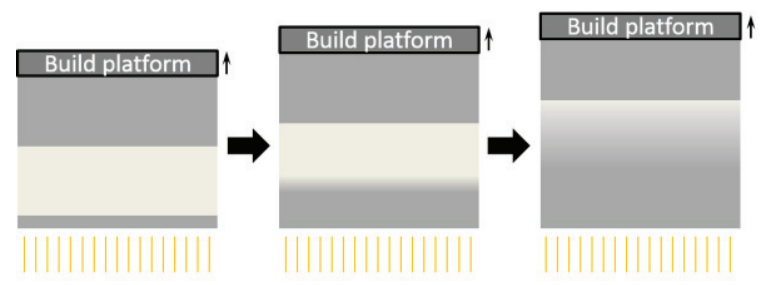

(b)

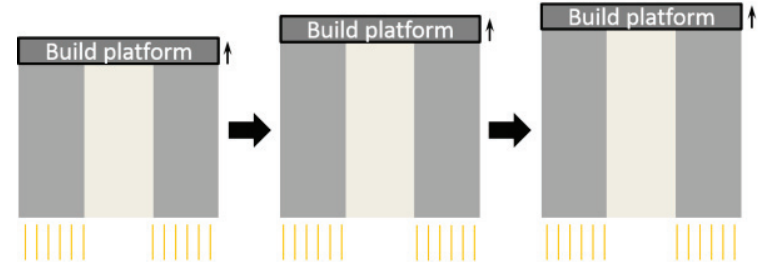

FIGURE 1. The layer-by-layer SL fabrication process for a simplified microfluidic channel built in the (a) horizontal (top row) and (b) vertical (bottom row) orientations.

During the SL DLP process, light is projected up through a transparent build tray, that contains the resin material, on to a build platform whereby it cures the resin in place. After the first layer is formed the build platform moves a set amount (defined by the set layer size) in the $\mathrm{z}$ direction and the process is repeated. One of the main drawbacks of the process occurs due to the leakage of light into previously processed 2D layers as subsequent layers are formed during the build process. This is depicted in Fig 1, and occurs as there is little control over the penetration depth of the curing light. This effect has been dubbed the back-side effect [2], however we will refer to it as the overcuring effect here. The problem resulting from overcuring [2] is that curing light from subsequent layers tends to cure the liquid resin inplace in internal structures. This is a major problem when building internal structures which require high dimensional accuracy such as microfluidic channel networks.

Two approaches in controlling the depth of cure have been explored in the literature. Most recently Tumbleston et al. [9] made improvements to SL DLP hardware by introducing an oxygen permeable build platform which inhibits polymerization and enables 'layerless' fabrication at faster build speeds. A second more widely researched approach involves altering the SL resin material with a chemical light absorber [6-8]. In this work we develop a third approach in mitigating for the overcuring effect by simulating the process and predicting the level of overcuring likely to occur.

\section{MATERIALS AND METHODS}

Parts were produced using an SL DLP 3D printer (Asiga Pico Plus 27). This particular model has a resolution quoted of $27 \mu \mathrm{m}$ in the X-Y plane. The z-layer thickness can be adjusted in $1 \mu \mathrm{m}$ increments with a minimum step size of $1 \mu \mathrm{m}$ and a maximum of $150 \mu \mathrm{m}$, however the maximum step size is limited by the resin material. In this study we use a Z-layer thickness of $50 \mu \mathrm{m}$. The 3D printer in this case operates at a curing wavelength just inside the UV spectrum at $405 \mathrm{~nm}$. Photocurable resins for SL 3D printing typically comprise one or more monomer materials combined with a photoinitiator and an optical light absorber. Here, we use a commercially available clear photocurable SL resin, PlasClear v2 (Asiga), that cures at $405 \mathrm{~nm}$. This resin yields a material with good mechanical and chemical properties and is ideal for microfluidic applications. Simple open channels were designed using Solidworks (Dassault Systems), an example of one of the parts is shown in Fig 2.

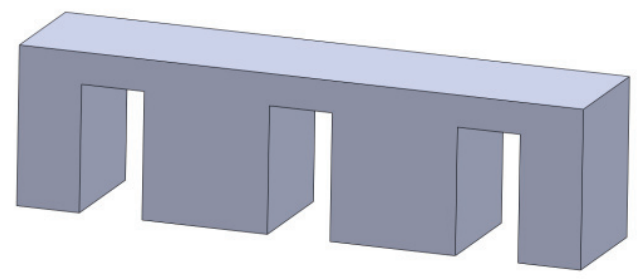

FIGURE 2. Schematic of the open channel design used in this study. The channels were printed with the open side in contact with the $3 \mathrm{D}$ printer build platform. 
Channels were built in triplicate with a varying number of enclosing layers above the channel $(5,10,15$, and 20 layers respectively). This enabled the measurement of the effects of overcuring due to light leakage from subsequent build layers. After production, the parts were rinsed with isopropyl alcohol (IPA) (Sigma Aldrich, IE) to remove any unsolidified resin and then immersed in a bath of IPA and sonicated for $\sim 5$ minutes. Care was taken during the rinse procedure to ensure that any unsolidified resin material was removed from internal channels. After sonication the parts were dried using pressurised air. Experimental dimensional measurements of the 3D printed channels was carried out using a Keyence VHX2000E 3D digital microscope with a 350x objective lens.

\section{Mathematical Model}

The simulated model for resin curing used in this work is based on the mathematical model developed previously by Gong et al. [7]. This model is expanded to two and three dimensions in the current work, for further detail on the derivation of the equation refer to reference [7]. In brief, the SL process can be modeled using the absorption coefficient $\alpha\left(\mu \mathrm{m}^{-1}\right)$ of the photopolymerisable resin to predict how deep the incident curing light will travel into the resin and thus predict the thickness of the final cured layer. This prediction is made using Beer's law where we define the characteristic light penetration depth as $h_{a}=1 / \alpha$ thus the equation for the optical irradiance with depth ' $\mathrm{z}$ ' is given in units of $\mathrm{W} \mathrm{cm}^{-2}$ by the equation:

$$
I(z)=I_{0} e^{-z / h_{a}}
$$

Where $I_{0}$ is the optical irradiance at a depth $z=0$. For the Asiga 3D printer this value is the light source intensity $\left(25 \mathrm{~W} \mathrm{~cm}^{-2}\right)$. The corresponding energy dose, $D(z, t)\left(\mathrm{J} \mathrm{cm}^{-2}\right)$ for an exposure time of $t(\mathrm{~s})$ is:

$$
D(z, t)=t I(z)
$$

Applying this to the photopolymerization process we define $D_{C}$, the critical dose, as the amount of energy it takes to form a solid, or nearly solid material from the liquid resin. Then the critical dose at an arbitrary distance $z_{p}$ (the polymerization depth) for a polymerization exposure time $t_{p}$ is given by:

$$
D_{c}=t_{p} I_{0} e^{-z / h_{a}}
$$

The values $z_{p}$ and $t_{p}$ are material properties and are interlinked (i.e. a particular exposure time $t_{p}$ will yield a polymerization depth $z_{p}$ and vice versa). Using these relationships, we can define the critical time, $T_{c}$ for a particular resin as:

$$
T_{c}=\frac{D_{c}}{I_{0}}
$$

From this, the polymerization depth, $z_{p}$ is given by:

$$
z_{p}=h_{a} \ln \frac{t_{p}}{T_{c}}
$$

From equations (2) and (4) we create the basic model for normalized dose $\Omega(z, t)$ that we apply in our simulations which is given by:

$$
\begin{gathered}
\Omega(z, t)=\frac{D(z, t)}{D_{c}} \\
=\frac{t}{T_{c}} e^{-z / h_{a}}
\end{gathered}
$$

From this relationship we can see that solidification of the resin occurs when the dose $D(z, t)$ surpasses the critical dose $D_{C}$. Therefore, the resin material reaches solidification when $\Omega \geq 1$. Varying height $(z)$ in increments of $1 \mu \mathrm{m}$ and thus solving equation (7) for $\Omega$ we can model the polymerization gradient of the resin material for a single layer. The model is scaled up further to take into account the curing dose received when concurrent layers are formed. This is achieved through summation of the dose received at each $z$ level in space calculated for each layer throughout the process. The final applied formula taken from [7] is given by:

$$
\Omega(z, t)=\sum_{n=0}^{N-1} \delta_{n} \Omega_{n}
$$




$$
\Omega(z, t)=\sum_{n=0}^{N-1} \delta_{n} \frac{t}{T_{c}} e^{\frac{-\left[(n+1) z_{l}-z\right]}{h_{a}}}
$$

where $N$ is the number of Layers, and $z_{l}$ is the layer thickness (constant throughout the experiment). In areas where a microfluidic channel is present, the UV lamp is masked by the printer's Digital Micromirror Device (DMD) and the channel area does not receive a curing dose, this is taken into account in the simulation formula by the binary parameter $\delta$ which equivalent to zero if a channel is present and one otherwise.

In the current research this model is scaled up from the single dimension to a three-dimensional model programmed using the graphical programming language LabVIEW (National Instruments, TX, USA). In addition, a number of SL DLP 3D printing process parameters are factored into the model including, layer thickness, $x-y$ pixel size, and basic part parameters including total part and channel dimensions.

The current simulation is based on a basic microfluidic channel with a square cross-section and is based on a bottom-up SL DLP 3D printer (i.e. Asiga Pico Plus 27) where the curing light is projected through the bottom of the resin bath in the vertical direction. This configuration is typical of many DLP 3D printers currently on the market. The simulation software created here solves the basic model for normalized dose $\Omega$ in the vertical $z$ direction using equations 1-9 and by working recursively and solving the equation at $1 \mu \mathrm{m}$ steps in the $z$ direction for each layer $(\mathrm{N})$ and for each pixel value $(27 \mu \mathrm{m})$ in $x$ and $y$ directions defined in the model space. In this work we are concerned with the vertical effects of the light projection, thus ignoring any minimal dispersion of the curing light in the horizontal direction.

\section{RESULTS AND DISCUSSION}

Simulations based on the mathematical model were produced using the printing parameters of the Asiga 3D printer with a slice thickness $\left(z_{l}\right)$ of $50 \mu \mathrm{m}$. Experimental models were then produced under the same conditions using the 3D printer. Figure 3 shows an example of the raw simulation output for a rectangular channel.

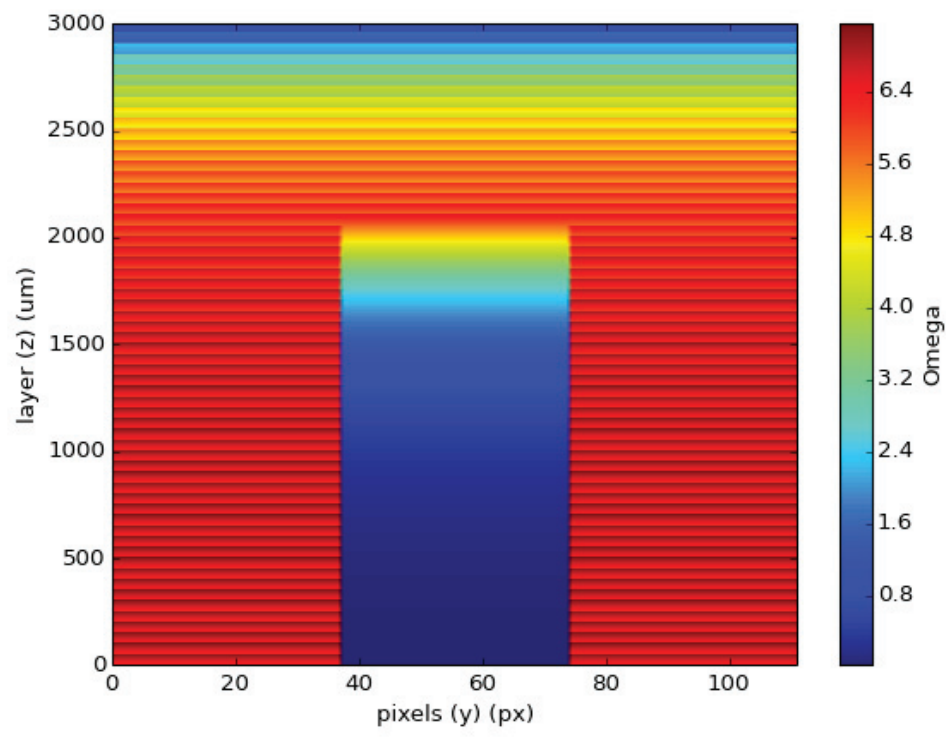

FIGURE 3. MP $\mu \mathrm{SL}$ simulation of a simple rectangular channel with dimensions 37 pixels (width) by $2000 \mu \mathrm{m}$ (height) in a block of solid material 111 pixels (width) by $3000 \mu \mathrm{m}$ (height). Layer thickness $z l=50 \mu \mathrm{m}$ was used throughout the simulation with values of $h_{a}$ and $T_{c}$ of $386 \mu \mathrm{m}$ and $2.3 \mathrm{~s}$ respectively. Total number of layers $\mathrm{N}=40$ layers.

Horizontal lines in the model are indicative of each individual $50 \mu \mathrm{m}$ layer, occurring as a result of the steep gradient change in light dose between the front $\left(\Omega_{\text {front }}\right)$ and back $\left(\Omega_{\text {back }}\right)$ of each layer. Values for $\Omega$ were in the range $\sim 0.03-7.15$ with values $\geq 1$ indicative of solid polymer material while values $<1$ indicate uncured liquid polymer resin. Using $\Omega=1$ as the polymerization point, a binary model was generated and the resulting channel height was calculated and compared to experimental results. This is a simplified assumption as the conversion from liquid to solid 
is defined by a gradient rather than a step change, however it serves as an appropriate indication of the solid/liquid boundary when compared with experimental values as shown in Fig. 4 below.

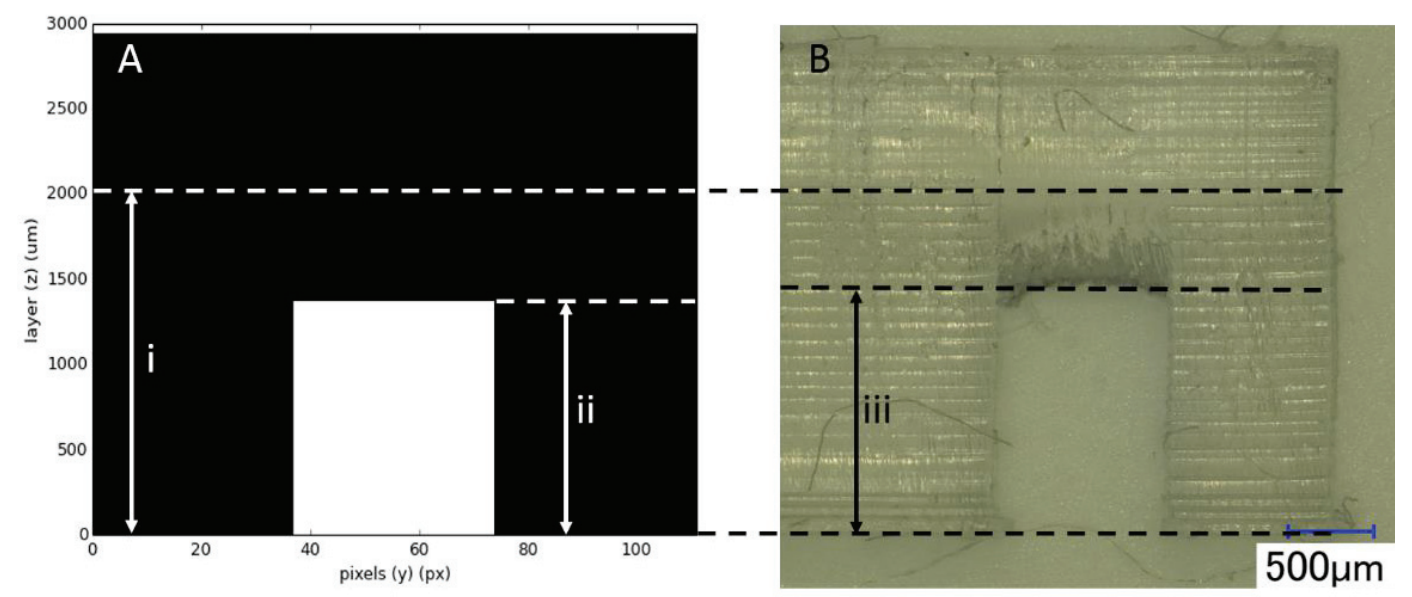

FIGURE 4. (A) Binary model extracted from the raw simulation data for a rectangular channel, and (B) the 3D printed part. Measurements shown are representative of the input channel height taken from the 3D CAD file (i), the simulated channel height given by the model (ii), and the actual channel height of the 3D printed part (iii).

For the binary model (Fig. 4 (A)) the simulated raw data (Fig. 3) was put through a filter to convert values of $\Omega$ above and below 1 to black and white respectively, where black values are analogous to a fully solidified part. As demonstrated in Fig. 4, simulation of the process enables the user to predict the level of overcuring in the microfluidic channel which is a function of the material properties $\left(h_{a}\right.$ and $\left.T_{c}\right)$, the experimental conditions (optical irradiance $\left(I_{0}\right)$, curing time $(t)$ ), and the design parameters (part orientation, slice thickness $\left(z_{l}\right)$ ). The level of overcuring present is also largely dependent on the number of enclosing layers above the channel. This effect is demonstrated in Fig. 5 below.

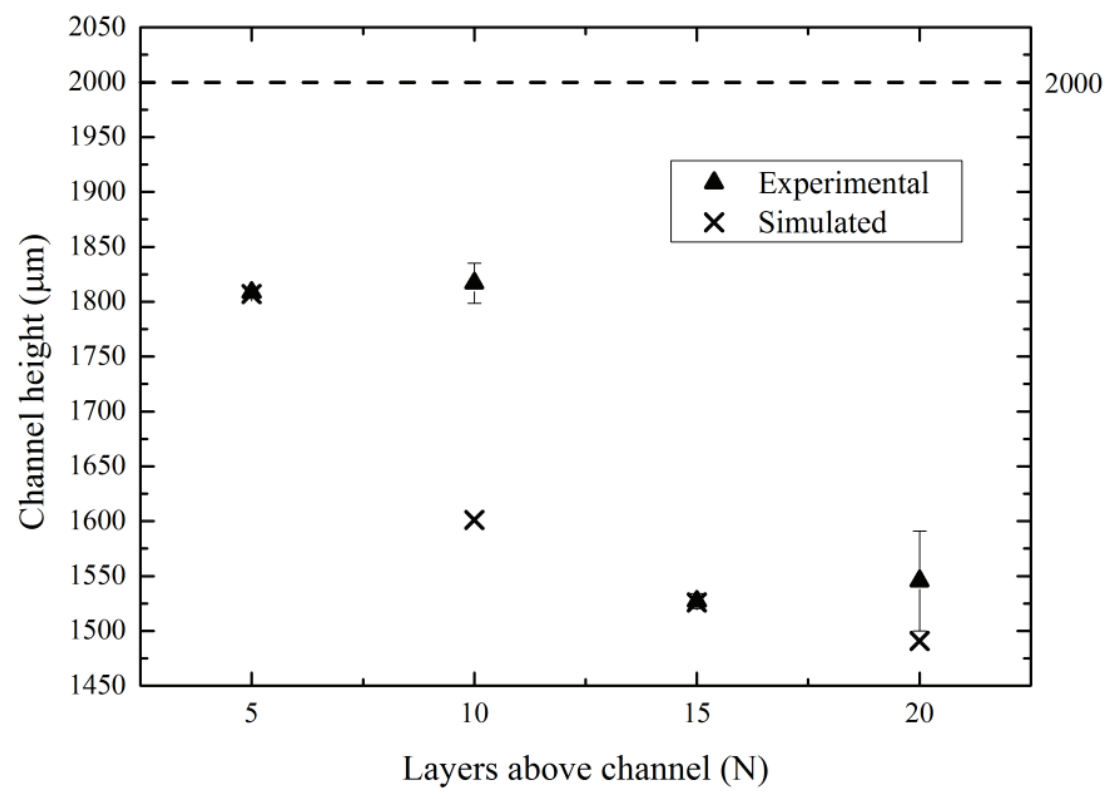

FIGURE 5. Experimental and simulated results for a $2000 \mu \mathrm{m}$ high microfluidic channel produced using MP $\mu$ SL 3D printing with varying number of enclosing layers above the channel. All channels have input dimensions of $2000 \mu \mathrm{m}$ height by $1000 \mu \mathrm{m}$ width. The simulation parameters $h_{a}$ and $T_{c}$ used were set as $386 \mu \mathrm{m}$ and $3.1 \mathrm{~s}$ respectively; error bars are standard deviation, $\mathrm{N}=500$. 
Figure 5 shows a strong correlation between simulated results and experimental measurements with values for 5 and 10 layers matching almost exactly. There is only one experimental outlier at 10 layers above the channel that does not follow the trend shown here which could be caused by experimental inconsistencies between resin material batches or by the presence of some already solidified material in the build tray prior to printing. Future work will focus toward scaling up this model to include other channel shapes and longer channels generating further comparisons between experimental and simulated results.

\section{CONCLUSIONS}

Using the model developed in this work it is possible to alter the 3D CAD file to mitigate for the effects of overcuring inherent in SL 3D printing processes. This presents a new approach to solving this problem while building on previous research which focused mainly on improvements in hardware $[8,9]$ and resin chemistry $[6,7]$. Future work will include the creation of an STL file modification program. It is envisioned that this program will improve the accuracy and reproducibility of internal structures produced using SL 3D printing methods and enable the creation of complex features, such as microfluidic networks, that were previously unrealizable.

\section{ACKNOWLEDGMENTS}

Financial support from Science Foundation Ireland (Grant Nos. 12/IA/1576 and 12/RC/2289) and the Naughton Foundation (Naughton Graduate Fellowship) is gratefully acknowledged.

\section{REFERENCES}

[1] G. Comina, A. Suska, and D. Filippini, "Low cost lab-on-a-chip prototyping with a consumer grade 3D printer.," Lab Chip, vol. 14, no. 16, pp. 2978-82, Aug. 2014.

[2] A. I. Shallan, P. Smejkal, M. Corban, R. M. Guijt, and M. C. Breadmore, "Cost-Effective ThreeDimensional Printing of Visibly Transparent Microchips within Minutes," Anal. Chem., vol. 86, no. 6, pp. 3124-3130, Mar. 2014.

[3] P. F. O’Neill, A. Ben Azouz, M. Vázquez, J. Liu, S. Marczak, Z. Slouka, H. C. Chang, D. Diamond, and D. Brabazon, "Advances in three-dimensional rapid prototyping of microfluidic devices for biological applications," Biomicrofluidics, vol. 8, no. 5, p. 52112, Sep. 2014.

[4] S. Waheed, J. M. Cabot, N. P. Macdonald, T. Lewis, R. M. Guijt, B. Paull, and M. C. Breadmore, "3D printed microfluidic devices: enablers and barriers," Lab Chip, vol. 16, no. 11, pp. 1993-2013, 2016.

[5] S. Sandron, B. Heery, V. Gupta, D. a Collins, E. P. Nesterenko, P. N. Nesterenko, M. Talebi, S. Beirne, F. Thompson, G. G. Wallace, D. Brabazon, F. Regan, and B. Paull, "3D printed metal columns for capillary liquid chromatography," Analyst, vol. 139, no. 24, pp. 6343-6347, Sep. 2014.

[6] J. Choi, R. B. Wicker, S. Cho, C. Ha, and S. Lee, "Cure depth control for complex 3D microstructure fabrication in dynamic mask projection microstereolithography," Rapid Prototyp. J., vol. 15, no. 1, pp. 5970, 2009.

[7] H. Gong, M. Beauchamp, S. Perry, A. T. Woolley, and G. Nordin, "Optical Approach to Resin Formulation for 3D Printed Microfluidics," RSC Adv., vol. 5, no. m, pp. 106621-106632, 2015.

[8] R. Janusziewicz, J. R. Tumbleston, A. L. Quintanilla, S. J. Mecham, and J. M. DeSimone, "Layerless fabrication with continuous liquid interface production," Proc. Natl. Acad. Sci., vol. 2016, p. 201605271, 2016.

[9] J. R. Tumbleston, D. Shirvanyants, N. Ermoshkin, R. Janusziewicz, A. R. Johnson, D. Kelly, K. Chen, R. Pinschmidt, J. P. Rolland, A. Ermoshkin, E. T. Samulski, and J. M. DeSimone, "Continuous liquid interface production of 3D objects," Science (80-. )., vol. 347, no. 6228, pp. 1349-1352, Mar. 2015.

[10] A. J. Capel, S. Edmondson, S. D. R. Christie, R. D. Goodridge, R. J. Bibb, and M. Thurstans, "Design and additive manufacture for flow chemistry.," Lab Chip, vol. 13, no. 23, pp. 4583-90, Dec. 2013. 